archives-ouvertes

\title{
Cauliflower fractal forms arise from perturbations of floral gene networks
}

\author{
Eugenio Azpeitia, Gabrielle Tichtinsky, Marie Le Masson, Antonio \\ Serrano-Mislata, Jérémy Lucas, Veronica Gregis, Carlos Gimenez, Nathanaël \\ Prunet, Etienne Farcot, Martin Kater, et al.
}

\section{To cite this version:}

Eugenio Azpeitia, Gabrielle Tichtinsky, Marie Le Masson, Antonio Serrano-Mislata, Jérémy Lucas, et al.. Cauliflower fractal forms arise from perturbations of floral gene networks. Science, American Association for the Advancement of Science, 2021, 373 (6551), pp.192-197. 10.1126/science.abg5999 . hal-03291136

\section{HAL Id: hal-03291136 \\ https://hal.archives-ouvertes.fr/hal-03291136}

Submitted on 21 Jul 2021

HAL is a multi-disciplinary open access archive for the deposit and dissemination of scientific research documents, whether they are published or not. The documents may come from teaching and research institutions in France or abroad, or from public or private research centers.
L'archive ouverte pluridisciplinaire HAL, est destinée au dépôt et à la diffusion de documents scientifiques de niveau recherche, publiés ou non, émanant des établissements d'enseignement et de recherche français ou étrangers, des laboratoires publics ou privés. 
2 Cauliflower fractal forms arise from perturbations of floral gene networks

5 Eugenio Azpeitia ${ }^{1, \$}$, Gabrielle Tichtinsky ${ }^{2}$, Marie Le Masson ${ }^{2}$, Antonio Serrano-Mislata ${ }^{3}$, Jérémy 6 Lucas $^{2}$, Veronica Gregis ${ }^{4}$, Carlos Gimenez ${ }^{3}$, Nathanaël Prunet ${ }^{5}$, Etienne Farcot ${ }^{6}$, Martin

7 M.Kater ${ }^{4}$, Desmond Bradley ${ }^{7}$, Francisco Madueño ${ }^{3}$, Christophe Godin $^{1, *}$, Francois Parcy ${ }^{2, *}$

9 1: Laboratoire de Reproduction et Développement des Plantes, Univ. Lyon, ENS de Lyon, UCB

10 Lyon 1, CNRS, INRAE, Inria, 46 allée d'Italie, F-69364, Lyon, France

11 2: Laboratoire Physiologie Cellulaire et Végétale, Univ. Grenoble Alpes, CNRS, CEA, INRAE, 12 IRIG-DBSCI-LPCV, 17 avenue des martyrs, F-38054, Grenoble, France

13 3: Instituto de Biología Molecular y Celular de Plantas (IBMCP), Consejo Superior de 14 Investigaciones Científicas (CSIC) - Universidad Politécnica de Valencia (UPV), 46022 15 Valencia, Spain.

16 4: Dipartimento di Bioscienze, Università degli Studi di Milano, Via Celoria 26, 20133 Milan, 17 Italy

18 5: Division of Biology and Biological Engineering, California Institute of Technology, $1200 \mathrm{E}$.

19 California Blvd., Pasadena, CA 91125, USA and Department of Molecular, Cell and 20 Developmental Biology, University of California, Los Angeles, 610 Charles E. Young dr. S., Los 21 Angeles, CA 90095, USA

22 6: School of Mathematical Sciences, University of Nottingham, NG7 2RD, United Kingdom.

23 7: Department of Cell and Developmental Biology, John Innes Centre, NR4 7UH Norwich NR4 $247 \mathrm{UH}$, United Kingdom.

25 †: Present address, Centro de Ciencias Matemáticas, Universidad Nacional Autónoma de 26 México, Morelia, México

27 *: Co-corresponding authors. 


\section{One Sentence Summary}

32 The molecular making of cauliflowers

\section{Abstract}

Throughout development, plant meristems regularly produce organs in defined spiral, opposite or whorl patterns, called phyllotaxis. Cauliflowers present an unusual phyllotaxis with a multitude of spirals nested over a wide range of scales. How such a fractal self-similar organization emerges from developmental mechanisms has remained elusive. Combining experimental analyses in Arabidopsis thaliana cauliflower-like mutant with modeling, we found that curd selfsimilarity arises because the meristems fail to form flowers but keep the "memory" of their transient passage in a floral state. Additional mutations affecting meristem growth can induce the

43 production of conical phyllotactic structures reminiscent of the conspicuous fractal Romanesco

44 shape. This study reveals how fractal-like forms may emerge from the combination of key, 45 defined perturbations of floral developmental programs and growth dynamics.

\section{Main Text}

Above-ground plant architectures arise from activity of shoot apical meristems (SAM), which are pools of stem cells that give rise to organs such as leaves, shoots or flowers. The arrangement of organs on stems is termed phyllotaxis. Plants with a spiral phyllotaxis usually form two families of organ spirals, visible on compact structures such as flower heads, pine cones or cacti

53 (Fig. 1a-c). These two families of spirals turn in opposite directions, and come in two consecutive numbers of the Fibonacci series (Fig. 1a) (1). In cauliflowers, spiral families are visible not only at one but at several scales (Fig. 1d-f). This self-similar organization culminates

56 in the Romanesco cultivar where the spirals appear in relief due to their conical shape at all scales, a geometrical feature conferring the whole curd a marked fractal-like aspect (Fig. 1g).

59 Cauliflowers (Brassica oleracea var. botrytis) were domesticated from cabbages (2). The

60 cauliflower inflorescence (the shoot bearing flowers) takes a curd shape because each emerging 
61 flower primordia never matures to the floral stage but instead generates more curd-shaped 62 inflorescences $(2,3)$. In B. oleracea, the genetic modifications causing curd development are still 63 debated and likely affect multiple genes (2-5). However, cauliflower-like structures also exist in 64 the model brassicaceae Arabidopsis thaliana and are caused by a double mutation in APETALA1 65 (AP1) and CAULIFLOWER (CAL) (Fig. 1h-i), two paralogous genes encoding MADS-box 66 transcription factors (TF) promoting floral development $(6,7)$. The Arabidopsis molecular 67 regulators governing the development of shoots and flowers have been largely identified (868 10)(Table S1). Network models based on these regulators have been proposed to explain wild69 type flower development (11-14). However, whether variants of these networks are able to 70 account for development of Arabidopsis apl cal curds is unknown.

72 To address this question, we first built a network of the main regulators involved in both flower 73 and curd development. Then, we embedded this network within a 3D computational model of 74 plant development to understand how mutations could transform wild-type (WT) inflorescences 75 into curds. 

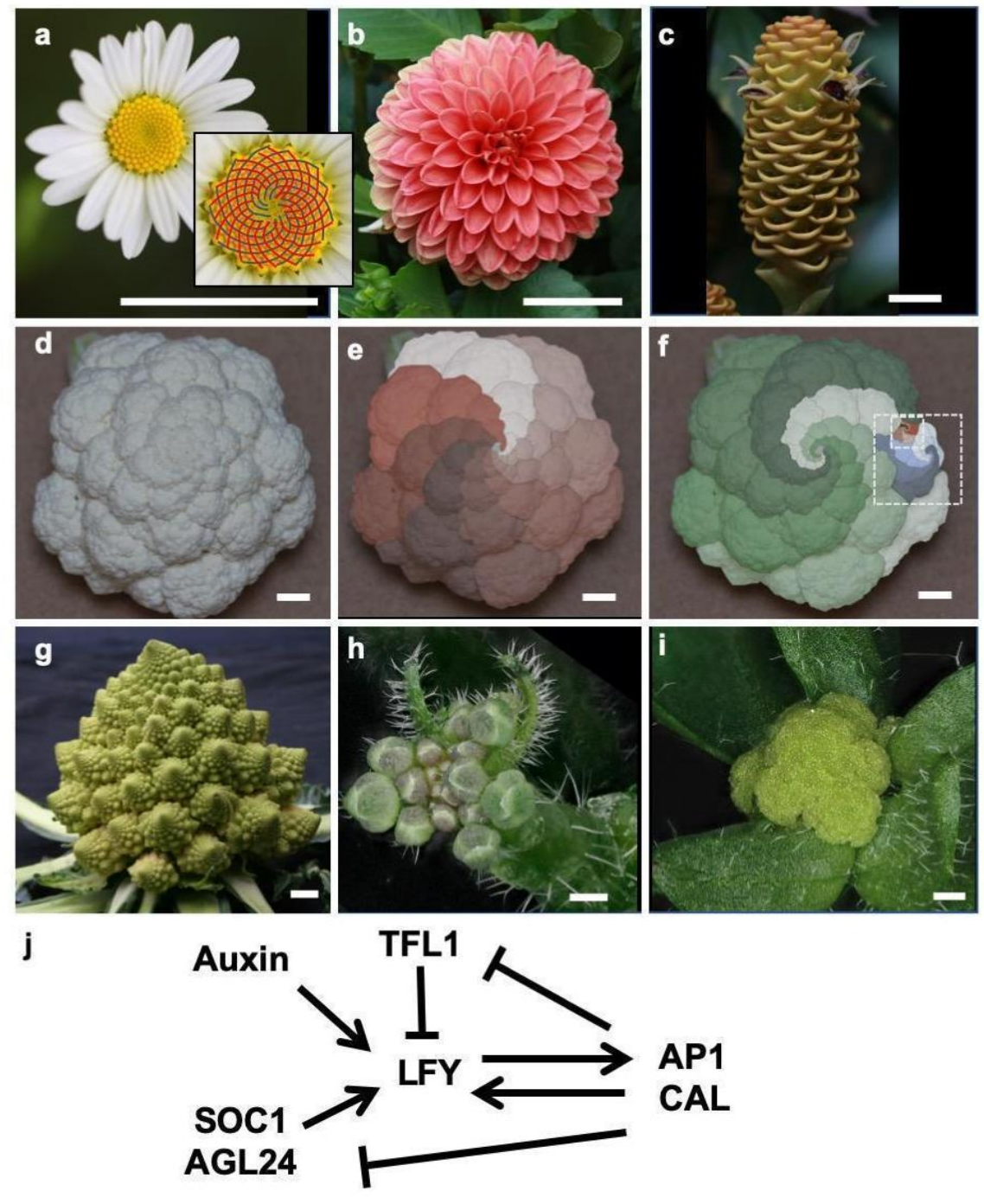

77 Figure 1: Illustrations of phyllotactic spirals on plant inflorescences

78 (a) Daisy capitulum: the two families of spirals are indicated in the close-up (13 blue spirals and 7921 red). (b) Dahlia composite flower (c) Zingiber inflorescence. (d-f) Brassica oleracea var.

80 botrytis cauliflower with (e) 8 counterclockwise (brown family) and (f) 5 clockwise (green

81 family) main spirals. Dashed rectangles show families of spirals nested over several scales (g)

82 Romanesco curd, (h) Arabidopsis wild-type inflorescence (h) and apl cal curd (i), Bar = $2 \mathrm{~cm}$ (a-

$83 \mathrm{~g}), 500 \mu \mathrm{m}(\mathrm{h}-\mathrm{i})$. (j) Interactions between major floral regulators; arrows depict activation

84 whereas barred lines indicate repression. 


\section{The genetic basis of cauliflower curds}

89 In Arabidopsis, flowers are initiated by the TF LEAFY (LFY) (Fig. 1j) (Table S1). LFY is 90 upregulated by the SUPPRESSOR-OF-OVEREXPRESSION-OF-CO 1 (SOC1) and 91 AGAMOUS-LIKE 24 (AGL24) MADS-box proteins (induced throughout the inflorescence

92 meristem by environmental and endogenous cues) and by auxin phytohormone maxima that 93 mark floral meristem initiation sites. $L F Y$ is expressed specifically in floral primordia because its 94 induction in the SAM is repressed by the TFL1 inflorescence identity protein. In the floral 95 primordium, LFY induces $A P 1$ and $C A L(A P 1 / C A L)$ that positively feedback on $L F Y$ and repress 96 both SOC1/AGL24 and TFL1, thereby stabilizing the floral fate of the new meristem. In the ap1 97 cal cauliflower mutant, the AP1/LFY positive feedback is absent and TFL1 is not repressed by 98 AP1/CAL in the nascent floral meristem. Consequently, young flower primordia cannot maintain $99 L F Y$ expression and start themselves expressing TFL1. As a result, they lose their floral identity 100 and become inflorescence meristems (6). Whereas TFL1 repression in nascent flower primordia 101 is well understood, the factors directly responsible for its upregulation in apl cal and 102 inflorescence meristems are unknown.

104 To complete our network, we thus searched for direct positive regulators of TFL1, other than 105 LFY (that induces TFL1 (15) but is not active in inflorescence meristems). TFL1 is indirectly 106 regulated by day length (16): in long days (LD) TFL1 is up-regulated by CONSTANS (CO) and 107 FT, two key upstream effectors of the LD pathway (11, 17-19) (Fig. S1). To search for direct 108 regulators, we examined SOC1 and AGL24 that act downstream of CO and FT in the LD 109 pathway (9). Loss- and gain-of-function experiments demonstrated that both SOC1 and AGL24 110 induce TFL1 (Fig. 2a-i) and Chromatin Immuno-Precipitation showed that these two TFs bind to 111 the TFL1 regions that regulate its expression in the SAM (20) (Fig. 2j-1). These regions were 112 sufficient to activate a TFL1 reporter construct by SOC1 and AGL24 in a transient assay (Fig. 113 2m-n) confirming that both MADS-box TFs are direct regulators of TFL1. Since XAANTAL2 114 (XAL2), a homolog of SOC1 and AGL24 also bound to and induced TFL1 (21), we aggregated 115 the activities of SOC1, AGL24 and XAL2 into a SAX proxy acting as TFL1 positive regulator 116 (Fig. 3a). 
118 We thus created the SALT network (for SAX, AP1/CAL, LFY, and TFL1; Fig. 3a) made of 119 these 4 regulator sets, auxin (22), and F, a flower inducing signal (a proxy for the FT florigen) 120 that increases when the plant ages or is exposed to flower-inducing environmental conditions 121 (23, 24). We also added a short-lived transient early Repressor of TFL1 (eREP), as a proxy for 122 TFL1 early repression in the young flower bud performed by the redundant activities of SOC1, 123 AGL24, SHORT VEGETATIVE PHASE, and SEPALLATA4 (25).

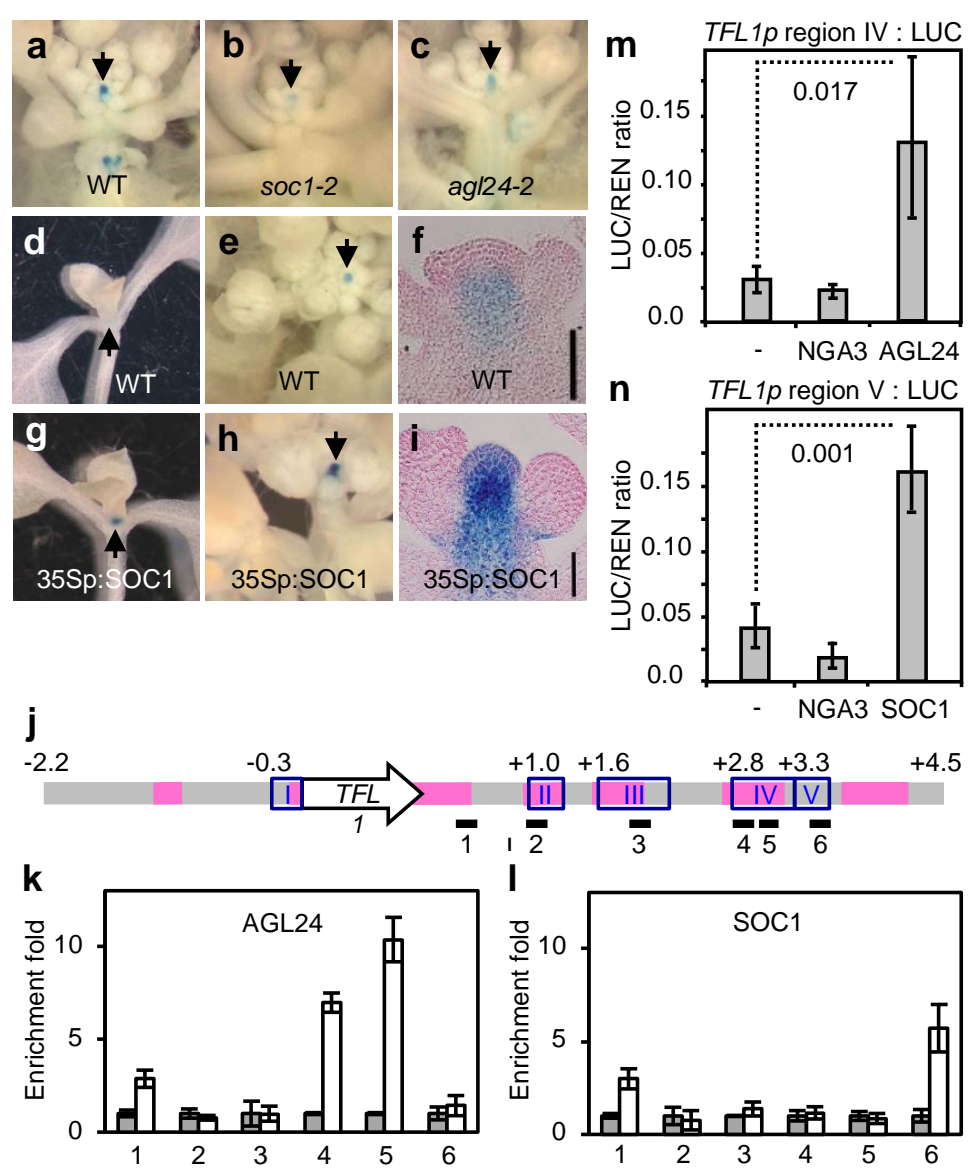

Fig. 2: AGL24 and SOC1 are direct positive regulators of TFL1.

126 (a-c), TFL1p:GUS activity in WT (a), soc1-2 (b) and agl24-2 (c) inflorescence apices. (d-i), 127 TFL1p:GUS activity (blue signal) in WT (d-f) and 35Sp:SOC1 (g-i) apices at vegetative (d,g) 128 and flowering (e,f,h,i) stages. (f-i), longitudinal sections through flowering shoots. Arrows mark 129 the SAM. Scale bars in (f) and (i), $40 \mu \mathrm{m}$. (j-1) Structure of TFL1 locus, with regions conserved 130 in Brassicaceae (pink lines), regulatory regions (20) (blue boxes I-V), and fragments used in 131 ChIP (black lines 1-6). ChIP experiments on plants expressing a tagged version of AGL24 (k, 
132 white bars) or the WT SOC1 protein (1, white bars) or on control plants (grey bars, see Material 133 and Methods), show that AGL24 binds region IV (k, fragments 4-5) and SOC1 region V (1, 134 fragment 6). A representative biological replicate is shown with the mean =/- SE for three 135 technical replicates. $(\mathrm{m}, \mathrm{n})$ Transient assays showing transactivation of the LUCIFERASE (LUC) 136 reporter driven by region IV (activation by 35Sp:AGL24) and region V (activation by 137 35Sp:SOC1). NGA3 is an unrelated TF used as negative control. Bars denote the mean and 138 standard deviation of three independent biological replicates. $P$ values are for the equality of 139 means (Student's t-test).

The steady states of the SALT network correspond to the gene expression patterns observed in 142 wild-type vegetative (low SALT values), inflorescence (high TFL1/SAX, low AP1/CAL/LFY) 143 and flower (low TFL1/SAX, high AP1/CAL/LFY) meristems (Fig. 3b,c, Fig. S2). Above an F 144 threshold value, the network generates a flower or an inflorescence state depending on $\mathrm{F}$ and 145 auxin values. Simulations of $t f l$, lfy, apl cal mutants produce expected outputs consistent with experimentally reported gene expressions $(6,16,26,27)$ (Fig. 3b, c). The simulated sax mutant

147 did not reach a floral state, consistent with the late flowering behavior of the soc1 agl24 double 148 mutant (28).

The modelled gene expression dynamics (Fig. 3d) illuminate the fundamental differences between WT and cauliflower meristems: in a WT flower primordium, F induces SAX. SAX and 152 auxin induce $L F Y$, that, together with $\mathrm{F}$, induce $A P 1 / C A L$. AP1 positively feeds back on $L F Y$ and 153 represses $S A X$ (Fig. 3d). TFL1 expression, that could be induced by SAX and LFY in early floral 154 stages, is constantly repressed, first by eREP and later by SAX plus AP1/CAL. High AP1/CAL 155 and LFY with low TFL1 and SAX expression stabilize the floral fate. In contrast, in the apl cal 156 flower primordia, the absence of AP1/CAL activity has two consequences: i) $L F Y$ expression is 157 upregulated only transiently since AP1/CAL positive feedback is missing (Fig. 3d) and ii) $S A X$ 158 genes are not repressed by AP1 and thus induce TFL1 in nascent flower meristems. TFL1 159 represses $L F Y$ even further and the meristem returns to a shoot meristem state (Fig. 3d). Note 160 that, the early LFY induction would likely be reinforced (while remaining transient) by 161 incorporating the recently discovered direct induction of LFY by the F partner protein FD (29). 162 The SALT model predicts that $S A X$ expression should extend over the entire cauliflower. We 
163 analyzed a SOC1-GFP reporter line and indeed observed expansion of its expression domain in 164 apl cal as compared to WT (Fig. 3e, f).

165
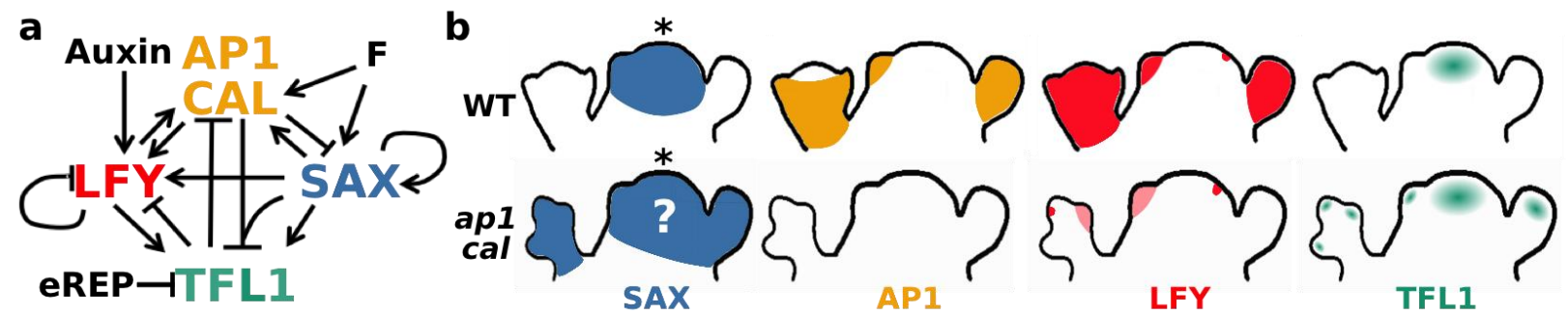

C
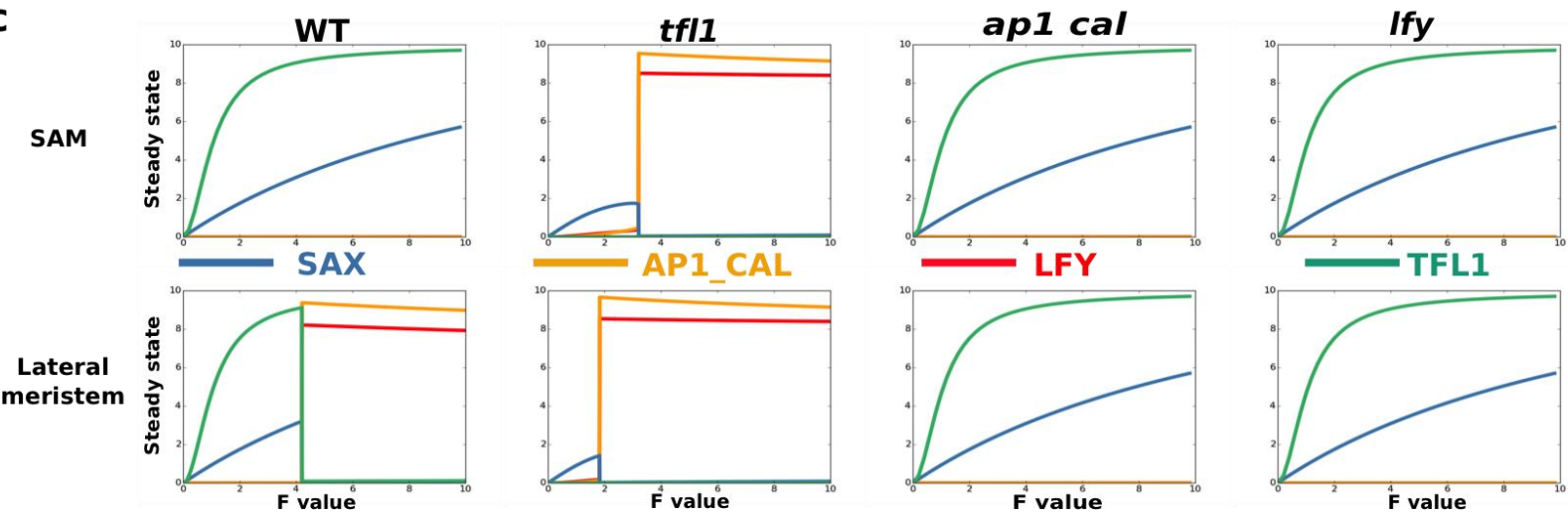

d

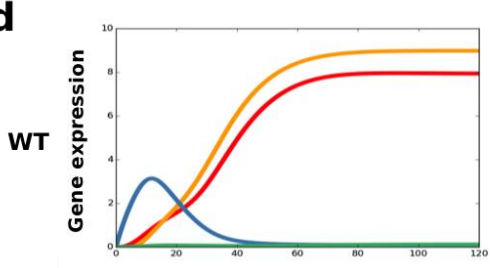

$\mathbf{f}$
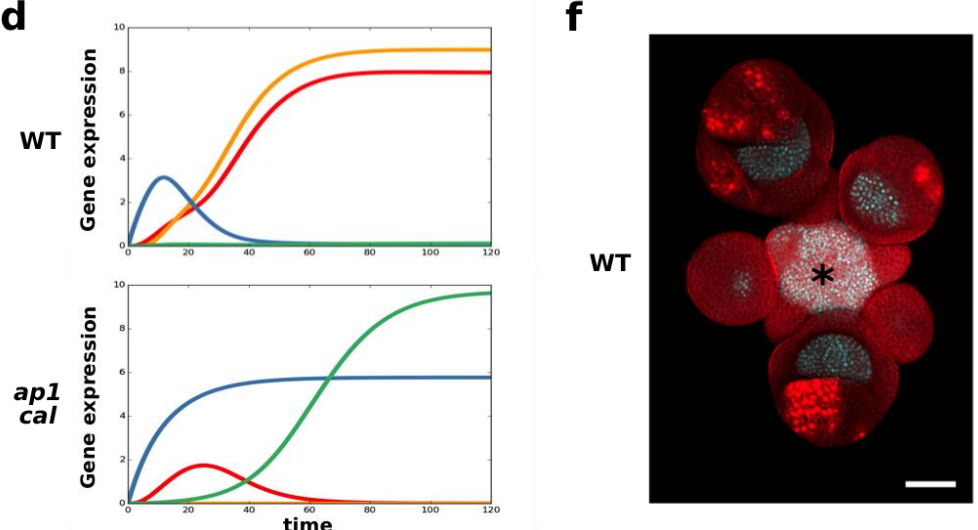

e

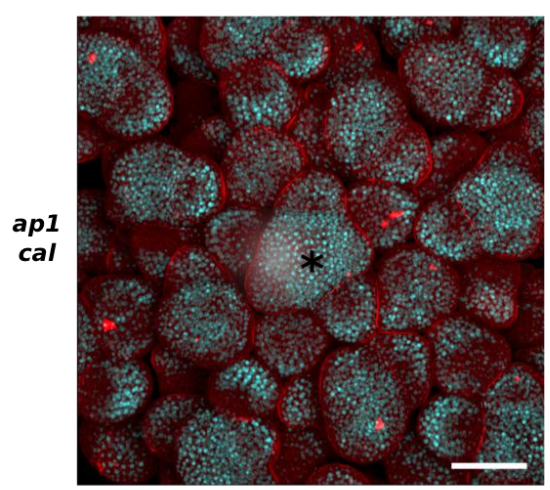

166

Fig. 3: SALT Gene Regulatory Network model and experimental validation.

168

(a) SALT GRN network structure (b) Known expression patterns of SAX, AP1/CAL, LFY, and

169 TFL1 in the SAM and lateral primordia of WT and apl cal mutant. The question mark indicates

170 a predicted expression pattern of the model. (c) WT, tfll, apl cal and lfy steady states of the

171 model at different F values in the SAM (low auxin) and in lateral meristems (high auxin). The

172 genetic identity predicted for WT and all mutant meristems correspond to the experimentally

173 observed phenotypes. (d) Temporal simulation of gene expression in lateral primordia with high 
174 F value. (e, f) Expression of the SOC1:GFP (white/light blue signal) reporter construct in WT (e)

175 and in the ap1-7 cal-1 mutant (f) inflorescences. Asterisks mark the SAM. Bar $=50 \mu \mathrm{m}$.

177 The SALT network thus recapitulates realistic gene expressions driving meristem fates. 178 However, a plant architecture does not only depend on meristem fates but also on 179 morphodynamic parameters including molecular thresholds for fate decisions, organ growth rate,

180 delay for meristems to start organ production and organ production rate which are independently 181 regulated. Plant inflorescence architecture thus emerges from the complex interaction between 182 the floral GRN and morphodynamic parameters. This is illustrated here by the lfy and apl cal 183 mutants that have the same GRN outputs (Fig. 3c) but markedly different architectures $(6,27)$.

184 To study how this interaction operates in Arabidopsis, we integrated the SALT GRN in a 3D 185 plant computational model implemented as an L-system (see Supplementary materials Modeling 186 Methods).

\section{A multi-scale model generates Arabidopsis cauliflower structures}

The 3D model is made of the 4 types of organs that shape plant above-ground architecture: meristems, internodes, leaves and flowers (Fig. 4a, Supplementary materials). Each meristem's identity (vegetative, inflorescence and floral) is determined by the GRN steady state, computed at each time step as a function of the meristem's previous state and external factors (auxin and

193 F). The GRN model is implemented as single compartment ordinary differential equations

194 (Supplementary materials Modeling Methods). We assume that the GRN dynamics is faster than growth and reaches its steady state within a time step. A set of growth rules defines meristem

196 production: a vegetative meristem produces a compressed stem (non-elongated internodes) with 197 rosette leaves; an inflorescence meristem produces an elongating internode, a cauline leaf and a new shoot meristem in the leaf axil; a floral meristem produces an internode terminating with a

199 flower meristem, devoid of bracts (leaf-like organs subtending flowers) since they are repressed 200 by LFY (6)). Each newly generated axillary meristem begins with maximal auxin level (22), 201 SAX/LFY/AP1/CAL values inherited from the parent meristem, together with a fraction of the 202 parent TFL1 value as, in the real plant, this non-cell autonomous protein is present in the 203 primordia region (30). To match the wild-type plant architecture, indeterminate meristems at 204 orders >2 (Fig. 4a) were kept quiescent, a likely effect of apical dominance (the inhibition of 
205 lateral meristem outgrowth) (Fig. S3a). The model also contains rules describing organ growth

206 dynamics (internode and leaf elongation, flower growth, organ production rate, growth initiation

207 delay). Simulated plants start with a single vegetative SAM and repeatedly produce new organs

208 according to the GRN, the morphodynamic rules and an input value of $\mathrm{F}$.

209 By adjusting the GRN and morphodynamic parameters within a range of plausible values 210 (Supplementary materials), we successfully calibrated the model to produce realistic 211 architectures for wild-type and lfy plants (Supplementary Movies 1-2), as well as for the tfll 212 mutant (Fig. 4b-d) and a non-flowering phenotype for the sax mutant. However, our simulations

213 could not generate a realistic apl cal mutant growing without bract/cauline leaves and displaying 214 high order meristems (Fig. S3a-b) suggesting that the cauliflower phenotype involves additional 215 regulations. We reasoned that laterally produced apl cal inflorescence meristems are different 216 from those produced in other genotypes as, according to our GRN, they have been transiently 217 exposed to LFY expression (Fig. 3d). Several pieces of evidence suggest that this transient LFY 218 expression, already known to repress bracts (6), could also contribute to high-order meristem 219 release. First, the outgrowth of otherwise inhibited axillary meristems in the rosette is stimulated 220 by ectopic expression of LFY (or a LFY allele) $(31,32)$. Second, it was established that the lfy 221 apl cal triple mutant does not form cauliflowers $(6)$ and we found that, in this mutant, the 222 number of high-order meristems is significantly reduced as compared to apl cal (Fig. S3d-h), 223 thus supporting our hypothesis.

224 We abstracted this critical molecular pathway, by introducing in the model a factor X 225 upregulated when LFY exceeds a minimal threshold level. Upregulated factor X releases high226 order meristem growth and suppresses the bract. This was sufficient to unlock the recursive 227 growth of lateral meristems and to generate the apl cal curd structure that arises from the 228 transient but irreversible exposure of meristems to the floral signal without any alteration of wild 229 type growth dynamics (Fig. 4e,h, Supplementary Movie 3). Overall, our work shows that the ap1 $230 \mathrm{cal}$ and lfy architectures are different (Fig. 3c) because the molecular histories of their 231 inflorescence meristems are different, thereby revealing the existence of a developmental 232 hysteresis. 

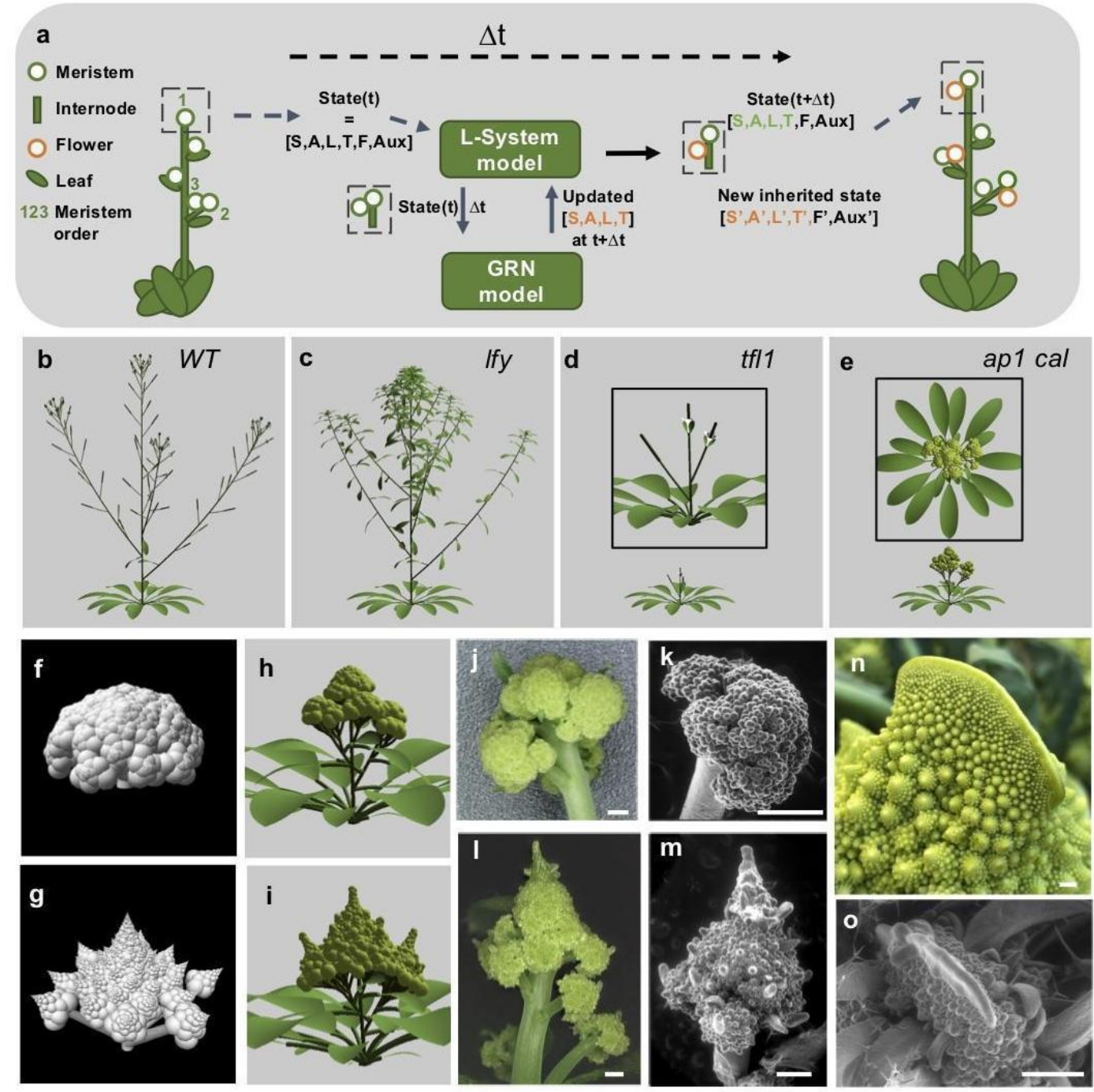

235 Fig. 4: Simulation and assessment of a GRN-based plant development model.

236 (a) Schematic representation of the multi-scale model of Arabidopsis development. Each 237 meristem state is composed of signal levels (auxin, F) and a GRN steady state. At time $t$, the 238 plant is made up of a collection of organs (left). At time $t+\Delta t$ (right) the model updates the signal 239 levels and GRN state in each meristem. The steady state defines the identity of the meristems 240 (vegetative, inflorescence or flower) used to compute meristem lateral productions. Green 241 numbers indicate meristem order (b-e). Plant morphologies obtained in the WT (b), lfy (c), tfll 242 (d) and apl cal (e) simulations. Simulated morphologies with constant (f,h) or increased 243 meristem size (g,i) in a simplified (f,g) and the Arabidopsis model (h,i). Light micrographs (j,1,n) 244 and s.e.m (k,m,o) of cauliflower structures in Arabidopsis apl cal (j, k), Arabidopsis apl cal 
$245 \operatorname{clv3}(1, \mathrm{~m}, \mathrm{o})$ and Romanesco $(\mathrm{n})$. Uninduced $A P 1: G R$ transgene is present in plants $\mathrm{j}$-m. Scale 246 bars $=500 \mu \mathrm{m}$.

\section{Growth dynamics define cauliflower and Romanesco curd structures}

249 Our work in Arabidopsis offers a conceptual framework to explain how inflorescence 250 architecture emerges from coupling a floral GRN to morphodynamic parameters. We wondered 251 whether modifications affecting components of this framework could also explain the 252 architecture of the cauliflowers that arose during domestication, namely the edible Brassica 253 oleracea (Bo) var. botrytis (Bob) and its Romanesco variant. Whether similar genetic defects as 254 in Arabidopsis are responsible for curd development in B. oleracea is still debated (4, 5). To 255 further investigate this point, we analysed RNA-seq data of Bob curds: we confirmed the 256 previously identified mutation in the BobCAL gene (Fig. S4a)(4, 5, 7) and observed that the two 257 AP1 paralogs (BobAP1- $a$ and BobAP1-c) are expressed at much lower levels than in cabbage (Bo 258 var. capitata) inflorescences (Fig. S4b). These functional proteins are induced with a delay only 259 when the cauliflower elongates and start forming normal flowers $(3,33)$. Comparing cauliflower 260 and cabbage sequences, we identified differences in binding sites for candidate regulators of 261 BoAP1 that could account for their delayed activation (Fig. S4d). The combination of BoCAL 262 inactivation and BobAPl-a/c expression delay (heterochrony due to $c$ is or trans mutations) thus 263 likely participates to Bob curd development. Similar to Arabidopsis apl cal, cauliflowers have 264 meristems of higher maximal order $(n \geq 7)$ than cabbages $(n=3-4)$ (Fig. S5). Nevertheless, the

265 development of single massive cauliflower curds is not the exact equivalent of the Arabidopsis 266 mutant $(3,5)$ and involves additional multifactorial alterations of morphodynamics parameters 267 (such as reduction of internode elongation and branches diameter increase).

269 The conical shapes appearing in Romanesco spirals at all scales (Fig. 1f) represent an additional 270 geometric variation obtained through domestication that seems to be associated with a change in 271 morphodynamic parameters. Indeed, several such parameters remain constant during cauliflower 272 development but vary in Romanesco (34): i) the plastochron, the time between two successive 273 meristem productions, ii) the number of visual spirals originating from a given meristem, iii) the 274 time (measured in number of plastochrons) needed before a lateral primordium starts producing 275 its own primordia (or lateral production onset delay), and iv) the size of the meristems. Whether 
276 some of these parameters are causal to the Romanesco phenotype remains unclear but

277 phyllotaxis studies $(1,35,36)$ indicate that the first three parameters are linked to the meristem

278 size: an augmentation of the size of the meristem central zone should decrease the plastochron,

279 which in turn increases the number of spirals, and the lateral production onset delay. We thus

280 hypothesized that passing from a constant to a decreasing plastochron in meristems could change 281 cauliflower into Romanesco morphologies. We first tested this in silico using a simplified, purely

282 geometric model of curd growth, independent from the Arabidopsis GRN and specific growth 283 dynamics (Supplementary materials). A decreasing plastochron was sufficient to produce 284 Romanesco shapes (Fig. 4g) whereas constant values of this parameter produce cauliflower 285 morphologies (Fig. 4f).

287 We then introduced the same change in the more complex GRN-based, Arabidopsis cauliflower architectural model, while keeping its organ growth dynamics as calibrated on the WT. Although not as complete as in the purely geometric model, the curd changed towards a "Romanesco-like" morphology with typical conical curd shapes (Fig. 4h, i). We then tested this hypothesis experimentally in Arabidopsis by altering the size of the meristem directly. We achieved this by introducing a mutation in the CLAVATA3 (CLV3) gene that controls meristem homeostasis and 293 induces an increase of the meristem central zone during growth $(37,38)$. As predicted by our analysis, introduction of a clv3 mutation in apl cal Arabidopsis mutant modified the curd shape, which lost its round morphology and acquired a more conical shape, with similar structures at different scales, features recognized as hallmarks of Romanesco curds (39) (Fig. 4l-m). Two additional pieces of evidence support the hypothesis that meristem homeostasis is perturbed in Romanesco curds: they occasionally show fasciation, a feature typical of meristem enlargement also observed in clv3 or apl cal clv3 mutants (Fig. 4n,o)(37). Moreover, the expression of CLV3

300 (and possibly two other genes acting in the same pathway)(38) are lower in Romanesco curds 301 than in cauliflowers (Fig. S6). Altogether, these observations establish that meristem size 302 regulates the final curd morphology through control of plastochron value.

304 These results reveal how fractal patterns can be generated through growth and developmental 305 networks that alter identities and meristem dynamics. Our data, GRN and growth models now 306 clarify the molecular and morphological changes over time by which meristems gain different 
307 identities to form the highly diverse and fascinating array of plant architectures found throughout 308 nature and crops.

309

310

311

312 1. C. Godin, C. Golé, S. Douady, Development. 147, dev165878 (2020).

313 2. C. F. Quiros, M. W. Farnham, in Genetics and Genomics of the Brassicaceae, R. Schmidt, 314 I. Bancroft, Eds. (Springer New York, 2011), pp. 261-289.

315 3. D. V. Duclos, T. Björkman, J. Exp. Bot. 59, 421-433 (2008).

316 4. L. B. Smith, G. J. King, Mol. Breeding. 6, 603-613 (2000).

317 5. N. Guo, S. Wang, L. Gao, Y. Liu, X. Wang, E. Lai, M. Duan, G. Wang, J. Li, M. Yang, 318 M. Zong, BMC Biology. 19, 93 (2021).

319 6. J. L. Bowman, J. Alvarez, D. Weigel, E. M. Meyerowitz, D. R. Smyth, Development. 119, $320 \quad 721(1993)$.

321 7. S. Kempin, B. Savidge, M. Yanofsky, Science. 267, 522 (1995).

322 8. G. Denay, H. Chahtane, G. Tichtinsky, F. Parcy, Curr. Opin. Plant Biol. 35, 15-22 (2017).

323 9. A. Pajoro , S. Biewers, E. Dougali, F. Leal Valentim, M. A. Mendes ,A. Porri, G. 324 Coupland, Y. Van de Peer, A.D. Van Dijk, L. Colombo, B. Davies, J. Exp. Bot. 65, 4731$325 \quad 4745$ (2014).

326 10. B. Thomson, F. Wellmer, Curr. Top. Dev. Biol. 131, 185-210 (2019).

327 11. K. E. Jaeger, N. Pullen, S. Lamzin, R. J. Morris, P. A. Wigge, Plant Cell. 25, 820 (2013).

328 12. C. Espinosa-Soto, P. Padilla-Longoria, E. R. Alvarez-Buylla, Plant Cell. 16, 2923-2939 329 (2004).

330 13. F. L.Valentim et al, PLOS ONE. 10, e0116973 (2015).

331 14. P. Prusinkiewicz, Y. Erasmus, B. Lane, L. D. Harder, E. Coen, Science. 316, 1452 (2007).

332 15. K. Goslin et al., Plant Physiol. 174, 1097 (2017).

333 16. C. Ferrandiz, Q. Gu, R. Martienssen, M. F. Yanofsky, Development. 127, 725 (2000).

334 17. D. Bradley, O. Ratcliffe, C. Vincent, R. Carpenter, E. Coen, Science. 275, 80 (1997).

335 18. X. Hou et al., Nat. Commun. 5, 4601 (2014).

336 19. S. K. Yoo et al., Plant Physiol. 139, 770 (2005). 
337 20. A. Serrano-Mislata et al., Development. 143, 3315 (2016).

338 21. R. V. Pérez-Ruiz et al., Mol. Plant. 8, 796-813 (2015).

339 22. D. Reinhardt et al., Nature. 426, 255-260.

340 23. P. A. Wigge, Curr. Biol. 21, R374-378 (2011).

341 24. J. Putterill, E. Varkonyi-Gasic, Curr. Opin. Plant Biol. 33, 77-82 (2016).

342 25. C. Liu et al., Dev. Cell. 24, 612-622 (2013).

343 26. O. J. Ratcliffe et al., Development. 125, 1609 (1998).

344 27. D. Weigel, J. Alvarez, D. R. Smyth, M. F. Yanofsky, E. M. Meyerowitz, Cell. 69, 843$345 \quad 859(1992)$.

346 28. S. D. Michaels et al., Plant J. 33, 867-874 (2003).

347 29. Y. Zhu et al., Nat. Commun. 11, 5118 (2020).

348 30. L. Conti, D. Bradley, Plant Cell. 19, 767 (2007).

349 31. H. Chahtane et al., Plant J. 74, 678-689 (2013).

$350 \quad 32 . \quad$ D. Weigel, O. Nilsson, Nature. 377, 495-500 (1995).

351 33. X. Sun et al., Environ. Exp. Bot.. 155, 742-750 (2018).

352 34. M. Kieffer, M. P. Fuller, A. J. Jellings, Planta. 206, 34-43 (1998).

353 35. S. Douady, Y. Couder, J. Theor. Biol. 178, 255-273 (1996).

354 36. Y. Refahi et al., eLife. 5, e14093 (2016).

355 37. J. C. Fletcher, Science. 283, 1911-1914 (1999).

356 38. M. Kitagawa, D. Jackson, Annu. Rev. Plant Biol. 70, 269-291 (2019).

357 39. L. E. Watts, Euphytica. 15, 111-115 (1966).

358 40. A. Maizel, D. Weigel, Plant J. 38, 164-171 (2004).

359 41. V. Grandi, V. Gregis, M. M. Kater, Plant J. 69, 881-893 (2012).

360 42. R. G. H. Immink et al., Plant Physiol. 160, 433 (2012).

361 43. I. Kardailsky, Science. 286, 1962-1965 (1999).

362 44. H. Lee, Genes Dev. 14, 2366-2376 (2000). 
363 45. V. Gregis, A. Sessa, C. Dorca-Fornell, M. M. Kater, Plant J. 60, 626-637 (2009).

364 46. H. Onouchi, M. I. Igeño, C. Périlleux, K. Graves, G. Coupland, Plant Cell. 12, 885-900 365 (2000).

366 47. M. Koornneef, C. J. Hanhart, J. H. van der Veen, Mol. Gen. Genet. 229, 57-66 (1991).

367 48. F. Wellmer, M. Alves-Ferreira, A. Dubois, J. L. Riechmann, E. M. Meyerowitz, PLoS 368 Genet. 2, e117 (2006).

369 49. R. A. Jefferson, T. A. Kavanagh, M. W. Bevan, EMBO J. 6, 3901-3907 (1987).

370 50. N. Bechtold, J. Ellis, G. Pelletier, C. R. Acad. Sci. Paris, Life Sci. 316, 1194-1199 (1993).

371 51. N. Bechtold, D. Bouchez, in Gene Transfer to Plants, I. Potrykus, G. Spangenberg, Eds. 372 (Springer Berlin Heidelberg, 1995) pp. 19-23.

373 52. M. Trigueros et al., Plant Cell. 21, 1394-1409 (2009).

374 53. I. Mitsuhara et al., Plant Cell Physiol. 37, 49-59 (1996).

375 54. R. Hellens et al., Plant Methods. 1, 13 (2005).

376 55. Z. Feng et al., Cell Res. 23, 1229-1232 (2013).

377 56. W. Yan, D. Chen, K. Kaufmann, Plant Methods. 12, 23 (2016).

378 57. S. Bensmihen et al., FEBS Lett. 561, 127-131 (2004).

379 58. S. J. Clough, A. F. Bent, Plant J. 16, 735-743 (1998).

380 59. N. Prunet, K. Duncan, J. Exp. Bot. 71, 2898-2909 (2020).

381 60. A. Sessions, D. Weigel, M. F. Yanofsky, Plant J. 20, 259-263 (1999).

382 61. D. Weigel, J. Glazebrook, Arabidopsis: a laboratory manual (Cold Spring Harbor

383 Laboratory Press, New York, 2002).

384 62. C. Dorca-Fornell et al., Plant J. 67, 1006-1017 (2011).

385 63. C. Belser et al., Nat. Plants. 4, 879-887 (2018).

386 64. J. Yu et al., BMC Genomics. 15, 3 (2014).

387 65. H. Lv et al., Sci Rep. 10, 12394 (2020).

388 66. O. Fornes et al., Nucleic Acids Res. 48, D87-D92 (2019).

389 67. E. Moyroud et al., Plant Cell. 23, 1293 (2011). 
390 68. B. H. Toyama, M. W. Hetzer, Nat. Rev. Mol. Cell Bio. 14, 55-61 (2013).

391 69. A. Jolma et al., Nature. 527, 384-388 (2015).

392 70. S. Legewie, H. Herzel, H. V. Westerhoff, N. Blüthgen, Mol. Syst. Biol. 4, 190 (2008).

393 71. S. Belikov, O. G. Berg, Ö. Wrange, Nucleic Acids Res. 44, 3045-3058 (2015).

394 72. S. Mangan, U. Alon, Proc. Natl. Acad. Sci. U.S.A. 100, 11980-11985 (2003).

395 73. P. Prusinkiewicz, A. Lindenmayer, The algorithmic beauty of plants (Springer-Verlag, $396 \quad$ New York, 1990).

397 74. F. Boudon, C. Pradal, T. Cokelaer, P. Prusinkiewicz, C. Godin, Front. Plant Sci. 3 (2012).

398 75. L. Mündermann, Y. Erasmus, B. Lane, E. Coen, P. Prusinkiewicz, Plant Physiol. 139, $399 \quad 960-968$ (2005).

400 76. C. M. Winter et al., Dev. Cell. 20, 430-443 (2011).

401 77. D. A. William et al., Proc. Natl. Acad. Sci. U.S.A. 101, 1775 (2004).

402 78. R. Benlloch et al., Plant J. 67, 1094-1102 (2011).

403 79. D. Wagner, R. W. M. Sablowski, E. M. Meyerowitz, Science. 285, 582 (1999).

404 80. F. Parcy, O. Nilsson, M. A. Busch, I. Lee, D. Weigel, Nature. 395, 561 (1998).

405 81. S. Hanano, K. Goto, Plant Cell. 23, 3172 (2011).

406 82. O. J. Ratcliffe, D. J. Bradley, E. S. Coen, Development. 126, 1109 (1999).

407 83. D. Goretti et al., Plant Physiol., 182 2081-2095 (2020).

408 84. J.-H. Jung, H.-J. Lee, J. Y. Ryu, C.-M. Park, Mol. Plant. 9, 1647-1659 (2016).

409 85. P. A. Wigge et al., Science. 309, 1056 (2005).

410 86. S. Collani, M. Neumann, L. Yant, M. Schmid, Plant Physiol. 180, 367-380 (2019).

411 87. M. Abe, Science. 309, 1052-1056 (2005).

412 88. P. Teper-Bamnolker, A. Samach, Plant Cell. 17, 2661-2675 (2005).

413 89. M. Romera-Branchat et al., Cell Rep. 31, 107717 (2020).

414 90. S.J. Liljegren, C. Gustafson-Brown, A. Pinyopich, G. S. Ditta, M. F. Yanofsky, Plant Cell. $415 \quad$ 11, 1007 (1999). 
416 91. K. Kaufmann et al., Science. 328, 85 (2010).

417 92. J. Lee, M. Oh, H. Park, I. Lee, Plant J. 55, 832-843 (2008).

418 93. J. Moon, H. Lee, M. Kim, I. Lee, Plant Cell Physiol. 46, 292-299 (2005).

419 94. C. Liu et al., Development. 135, 1481 (2008).

420 95. N. Yamaguchi et al., Dev Cell. 24, 271-282 (2013).

421 96. W. Li et al., Sci. Signal. 6, ra23 (2013).

422 97. O. Nilsson, I. Lee, M. A. Blázquez, D. Weigel, Genetics. 150, 403-410 (1998).

423 98. A. Serrano-Mislata et al., Plant Signal. Behav. 12, e1370164 (2017).

424 99. C. Liu et al., Development. 134, 1901 (2007).

425 100. H. Yu, T. Ito, F. Wellmer, E. M. Meyerowitz, Nat. Genet. 36, 157 (2004).

426 101. Z. Tao et al., Plant J. 70, 549-561 (2012).

427 102. H. Yu, Y. Xu, E. L. Tan, P. P. Kumar, Proc. Natl. Acad. Sci. U.S.A. 99, 16336-16341 $428 \quad$ (2002).

429 103. J.-W. Wang, B. Czech, D. Weigel, Cell. 138, 738-749 (2009).

430 104. S. D. Michaels, E. Himelblau, S. Y. Kim, F. M. Schomburg, R. M. Amasino, Plant $431 \quad$ Physiol. 137, 149-156 (2005).

432 105. I. Searle, Gene. Dev. 20, 898-912 (2006).

433 106. R. Borner et al., Plant J. 24, 591-599 (2000).

434 107. A. Samach, Science. 288, 1613-1616 (2000).

435 108. S. R. Hepworth, The EMBO J. 21, 4327-4337 (2002).

\section{Acknowledgments}

438 We thank Anne-Marie Chèvre, Richard Immink, Rüdiger Simon, Lars Ostergaard and Mariana 439 Benitez for advice, Teva Vernoux, Chloe Zubieta and Hicham Chahtane for proofreading and 440 useful feedback on the manuscript, Dominique Tardy, Eric Giraud, Renaud Dumas and Vincent 441 Martin (OBS, France) for providing cauliflower samples and Lydia Bousset Vaslin for images 442 and branch counting, Frédéric Boudon for help with L-Py, Richard Immink (Wageningen, 443 Netherlands), C. Ferrándiz (IBMCP; Spain), George Coupland (MPIPZ, Germany), Miguel 
444 Ángel Blázquez (IBMCP, Spain), Richard Amasino (UWM, USA) and the European 445 Arabidopsis Stock Centre for providing seeds, Vincent Berger (CEA/DRF) for the Keyence 446 microscope, Christine Lancelon-Pin (Plateau de microscopie électronique - ICMG. CERMAV447 CNRS) for SEM experiments.

449 Funding

450 This project received support from the INRAE Caulimodel project (FP and CG), Inria Project 451 Lab Morphogenetics (CG, EA and FP), the ANR BBSRC Flower model project (FP and CG), the 452 GRAL LabEX (ANR-10-LABX-49-01) with the frame of the CBH-EUR-GS (ANR-17-EURE453 0003), EU H2020 773875 ROMI project funding (CG), the Spanish Ministerio de Ciencia 454 Innovación and FEDER (grant no. PGC2018-099232-B-I00)(FM).

456 Author contributions

457 ChG and FP conceived the study

458 ChG, EA, EF performed the modelling

459 ASM, CaG, DB, FM, FP, GT, MK, MLM, VG designed and performed the plant experiments

460 NP performed the confocal imaging experiment

461 JL analysed the RNA-seq and genomic data

$462 \mathrm{ChG}, \mathrm{FP}$ and EA wrote the paper with the help of all authors

\section{Competing interests}

465 The authors declare no competing interests.

\section{Data and Materials Availability}

469 All data are in the main paper or the supplement.

471 All plant materials are available upon request.

472 The following secure token has been created to allow review of record GSE150627 while it

473 remains in private status: khkjgckmdtkhpgb.

474 All source codes to run the simulations are available as supplementary archive file (description 475 of installation and execution available as README.txt. 
476

477

478 Supplementary Materials:

479 Materials and Methods

$480 \quad$ Figures $\mathrm{S} 1$ to $\mathrm{S} 6$

481 Tables $\mathrm{S} 1$ toS3

482 Movies S1 to S3

483 Code archive file: Architecture-model.zip

484 References (41-108)

485 MDAR Reproducibility Checklist

486

487

488 